International Journal of Pure and Applied Mathematics

Volume 85 No. 4 2013, 679-689

ISSN: 1311-8080 (printed version); ISSN: 1314-3395 (on-line version)

url: http://www.ijpam.eu

doi: http://dx.doi.org/10.12732/ijpam.v85i4.5

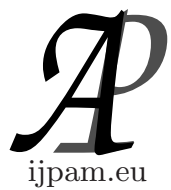

\title{
SOME NEW GENERALIZED DIFFERENCE SEQUENCE SPACES ON SEMINORMED SPACE DEFINED BY ORLICZ FUNCTION
}

\author{
M. Aiyub \\ Department of Mathematics \\ University of Bahrain \\ P.O. Box 32038, KINGDOM OF BAHRAIN
}

\begin{abstract}
In this paper, we define the sequence space $\ell_{M}\left(\Delta^{m}, u, p, q, s\right)$ on seminormed complex linear space by using an orlicz function.we give various properties and some inclusion relations involving $\ell_{M}\left(\Delta^{m}, u, p, q, s\right)$. This study generalized some results of Bektaş and Altin [1].
\end{abstract}

AMS Subject Classification: 40A05, 46A45

Key Words: Orlicz function, difference sequence, solid space, sequence algebra

\section{Introduction and Preliminaries}

Let $\ell_{\infty}, c$ and $c_{0}$ be the linear spaces of bounded, convergent and null sequence $x=\left(x_{k}\right)$ with complex number, respectively, normed by $\|x\|_{\infty}=\sup _{k}\left|x_{k}\right|$, where $k \in \mathbb{N}$. Throughout this paper $\omega(X), \ell_{\infty}(X), c(X)$ and $c_{0}(X)$ denote the class of all bounded, convergent and null $X$-valued sequences, where $(X, q)$ is a seminormed space, seminormed by q. The zero sequence is denoted by $\bar{\theta}=$ $(\theta, \theta \ldots \ldots$.$) , where \theta$ is the zero element of $X$. The spaces are seminormed spaces seminormed by $g(x)=\sup _{k \in \mathbb{N}} q\left(x_{k}\right)$. For $X=\mathbb{C}$, the set of complex numbers, these represent the above corresponding scalar valued sequence spaces.

The idea of difference sequence sets was introduced by Kizmaz [7] and this

Received: November 23, 2012

(c) 2013 Academic Publications, Ltd. url: www.acadpubl.eu 
subject was generalized by Et and Çolak [4]. After then the difference sequence spaces have been studied by various author such as Et [3], Et and Nurary [5], Malkowsky and Prasar [13], Mursaleen [14] Tripathy [18], [19], Tripathy et.al [20].

The study of orlicz sequence spaces was initiated with a certain specific purpose in Banach space theory. Indeed Lindberg [9] got interested in orlicz spaces in connection with finding Banach spaces with symmetric Schauder bases having complementary subspaces isomorphic to $c_{0}$ or $\ell_{\infty} \quad\left(1 \leq p_{k} \leq \infty\right)$.

Prashar and Choudhry [16] have introduced and discussed some properties of the four sequence spaces defined by using an orlicz function M, which generalized the sequence space $\ell_{M}$ and strongly summable sequence spaces $[C, 1, p],[C, 1, p]_{0}$ and $[C, 1, p]_{\infty}$. Later on different types of sequence spaces were introduced by using an orlicz function by Bektas and Altin [1] Tripathy [19], Tripathy et al.[20]. The orlicz sequence spaces are the special cases of orlicz spaces introduced in [8]. Orlicz sequence spaces find a number of useful application in theory of nonlinear integral equation. Where as the orlicz sequence spaces are the generalizations of $\ell_{p}$-spaces, the $\mathbf{L}_{p}$-spaces find themselves enveloped in orlicz spaces.

The main purpose of this paper is to introduce and study the sequence space $\ell_{M}\left(\Delta^{m}, u, p, q, s\right)$ which arises from the notion of generalized difference operator $\Delta^{m}$ and the concept of an orlicz function.

In this section, using the generalized difference operator $\Delta^{m}$ and concept of an orlicz function, we generalized the sequence space $\ell_{M}(p)$ which was introduced by Prashar and Choudhry [16].

The difference sequence space, $Z(\Delta)=\left\{x=\left(x_{k}\right): \Delta x \in Z\right\}$, where $X=\ell_{\infty}, c$ and $c_{0}$, were studied by Kizmaz [7]. The notion of difference sequence spaces was generalized by Et and Çolak [4] as follow

$$
Z\left(\Delta^{m}\right)=\left\{x=\left(x_{k}\right): \Delta^{m} x_{k} \in Z\right\}
$$

for $Z=\ell_{\infty}, c$ and $c_{0}$, where $m \in \mathbb{N}, \Delta^{m} x_{k}=\left(\Delta^{m-1} x_{k}-\Delta^{m-1} x_{k+1}\right)$ for all $k \in \mathbb{N}$.

The generalized difference operator has the following binomial representation,

$$
\Delta^{m} x_{k}=\sum_{v=0}^{m}(-1)^{v}\left[\begin{array}{c}
m \\
v
\end{array}\right] x_{k+v}, \text { for all } k \in \mathbb{N} .
$$

These sequence spaces are BK-spaces with the norm $\|x\|_{\Delta}=\sum_{i=1}^{m}\left|x_{i}\right|+$ $\left\|\Delta^{m} x\right\|_{\infty}$. 
An orlicz function is a function $M:[0, \infty) \rightarrow[0, \infty)$ which is continuous, non-decreasing and convex with $M(0)=0, M(x)>0$ for $x>0$ and $M(x) \rightarrow$ $\infty$, as $x \rightarrow \infty$, see [8]

If the convexity of orlicz function $M$ is replaced by $M(x+y) \leq M(x)+M(y)$ then this function is called modulus function introduced by Nakano [15] and further investigated by Ruckle [17], Maddox [11], Bilgin [2] and other.

Lindenstrauss and Tzafriri [10] defined the sequence space $\ell_{M}$ such as

$$
\ell_{M}=\left\{x \in \omega: \sum_{k=1}^{\infty} M\left(\frac{\left|x_{k}\right|}{\rho}\right)<\infty, \text { for some } \rho>0\right\} .
$$

The space $\ell_{M}$ with the norm $\left.\|x\|=\inf \left\{\rho>0: \sum_{k=1}^{\infty} M\left(\frac{\left|x_{k}\right|}{\rho}\right) \leq 1\right)\right\}$ becomes a Banach space which is called an orlicz sequence space. For $M(t)=t^{p}$, $1 \leq p \leq \infty$ the space $\ell_{M}$ is closely related to the sequence space $\ell_{p}$.

Definition 1. Let $\mathrm{X}$ be a complex linear with zero element $\theta$ and $(X, q)$ be the seminored space with norm $q$. By $\omega(X)$ we denote the linear space of all sequences and $x=\left(x_{k}\right)$ with $x_{k} \in X$ and the usual coordinate wise operations:

$$
\alpha x=\left(\alpha x_{k}\right) \text { and } x+y=\left(x_{k}+y_{k}\right)
$$

for each $\alpha \in \mathbb{C}$ where $\mathbb{C}$ denotes the set of complex numbers. If $\lambda=\left(\lambda_{k}\right)$ is a scalar sequence and $x \in \omega(X)$ then we shall write $\lambda x=\left(\lambda_{k} x_{k}\right)$. Let $\mathrm{U}$ be the set of all sequences $u=\left(u_{k}\right)$ such that $u_{k} \neq 0$ and complex for all $k=1,2,3 \ldots$ Let $p=\left(p_{k}\right)$ be sequence of positive real numbers and $M$ be an orlicz function. Given $u \in U$. Let $s \geq 0$. Then we define the sequence space.

$$
\begin{aligned}
& \ell_{M}\left(\Delta^{m}, u, p, q, s\right)= \\
& \quad\left\{x \in \omega(X): \sum_{k=1}^{\infty} k^{-s}\left[M\left(q\left(\frac{u_{k} \Delta^{m} x_{k}}{\rho}\right)\right)\right]^{p_{k}}<\infty, \text { for some } \rho>0\right\} .
\end{aligned}
$$

The following inequalities will be used throughout this paper. Let $p=\left(p_{k}\right)$ be a sequence of strictly positive real numbers with $0 \leq p_{k} \leq \sup _{p_{k}}=G$ and let $D=\max \left(1,2^{G-1}\right)$. Then for all $k$ and $a_{k}, b_{k} \in \mathbb{C}$, we have

$$
\left|a_{k}+b_{k}\right|^{p_{k}} \leq D\left\{\left|a_{k}\right|^{p_{k}}+\left|b_{k}\right|^{p_{k}}\right\}
$$

A sequence space $E$ is said to be solid (or normal) if $\left(\alpha_{k} x_{k}\right) \in E$ whenever $\left(x_{k}\right) \in E$ for all sequences $\left(\alpha_{k}\right)$ of scalar with $\left|\alpha_{k}\right| \leq 1$.

Remark 1.1. If $M$ is convex function and $M((0)=0$, then $M(\lambda x) \leq$ $\lambda M(x)$ for all $\lambda$ with $0 \leq \lambda \leq 1$. 


\section{Main Results}

Theorem 2.1. The sequence space $\ell_{M}\left(\Delta^{m}, u, p, q, s\right)$ is a linear space over the field $\mathbb{C}$ complex number.

Proof. Let $x, y \in \ell_{M}\left(\Delta^{m}, u, p, q, s\right)$ and $\alpha, \beta \in \mathbb{C}$. Then there exist some positive numbers $\rho_{1}, \rho_{2}$ such that

$$
\sum_{k=1}^{\infty} k^{-s}\left[M\left(q\left(\frac{u_{k} \Delta^{m} x_{k}}{\rho_{1}}\right)\right)\right]^{p_{k}}<\infty
$$

and

$$
\sum_{k=1}^{\infty} k^{-s}\left[M\left(q\left(\frac{u_{k} \Delta^{m} y_{k}}{\rho_{2}}\right)\right)\right]^{p_{k}}<\infty
$$

Define $\rho_{3}=\max \left(2|\alpha| \rho_{1}, 2|\beta| \rho_{2}\right)$. Since $M$ is non-decreasing and convex, $q$ is seminorm and $\Delta^{m}$ is linear we have

$$
\begin{gathered}
\sum_{k=1}^{\infty} k^{-s}\left[M\left(q\left(\frac{u_{k} \Delta^{m}\left(\alpha x_{k}+\beta y_{k}\right)}{\rho_{3}}\right)\right)\right]^{p_{k}} \\
\leq \sum_{k=1}^{\infty} k^{-s}\left[M\left(q\left(\frac{u_{k} \Delta^{m} \alpha x_{k}}{\rho_{3}}\right)\right)+q\left(\frac{u_{k} \Delta^{m} \beta y_{k}}{\rho_{3}}\right)\right]^{p_{k}} \\
\leq \sum_{k=1}^{\infty} \frac{1}{2^{p_{k}}} k^{-s}\left[M\left(q\left(\frac{u_{k} \Delta^{m} x_{k}}{\rho_{1}}\right)\right)+M\left(q\left(\frac{u_{k} \Delta^{m} y_{k}}{\rho_{2}}\right)\right)\right]^{p_{k}} \\
\leq \sum_{k=1}^{\infty} k^{-s}\left[M\left(q\left(\frac{u_{k} \Delta^{m} x_{k}}{\rho_{1}}\right)\right)+M\left(q\left(\frac{u_{k} \Delta^{m} y_{k}}{\rho_{2}}\right)\right)\right]^{p_{k}} \\
\leq D \sum_{k=1}^{\infty} k^{-s}\left[M\left(q\left(\frac{u_{k} \Delta^{m} x_{k}}{\rho_{1}}\right)\right)\right]^{p_{k}}+D \sum_{k=1}^{\infty} k^{-s}\left[M\left(q\left(\frac{u_{k} \Delta^{m} y_{k}}{\rho_{2}}\right)\right)\right]^{p_{k}}<\infty
\end{gathered}
$$

This proves that $\ell_{M}\left(\Delta^{m}, u, p, q, s\right)$ is linear.

Theorem 2.2. The sequence space $\ell_{M}\left(\Delta^{m}, u, p, q, s\right)$ is paranormed (not necessarily totaly paranormed) with

$$
g_{u}(x)=\inf \left\{\rho^{\frac{p_{n}}{H}}:\left(\sum_{k=1}^{\infty} k^{-s}\left[M\left(q\left(\frac{\Delta^{m} u_{k} x_{k}}{\rho}\right)\right)\right]^{p_{k}}\right)^{\frac{1}{H}} \leq 1 \quad n=1,2,3 . .\right\},
$$

where $H=\max \left(1, \sup _{k} p_{k}\right)$. 
Proof. Clearly $\left.g_{u}(x)=g_{u}(-x)\right)$. The subadditivity of $g_{u}$ follows from equation(2) on taking $\alpha=1$ and $\beta=1$. Since $q(\theta)=0$ and $M(0)=0$, we get $\inf \left\{\rho^{\frac{p_{n}}{H}}\right\}=0$ for $x=\theta$.

Finally, we prove that the scalar multiplication is continuous. Let $\lambda$ be any number.

By definition,

$$
g_{u}(\lambda x)=\inf \left\{\rho^{\frac{p_{n}}{H}}:\left(\sum_{k=1}^{\infty} k^{-s}\left[M\left(q\left(\frac{\Delta^{m} \lambda u_{k} x_{k}}{\rho}\right)\right)\right]^{p_{k}}\right)^{\frac{1}{H}} \leq 1 \quad n=1,2,3 . .\right\}
$$

Then

$$
g_{u}(\lambda x)=\inf \left\{(\lambda r)^{\frac{p_{n}}{H}}:\left(\sum_{k=1}^{\infty} k^{-s}\left[M\left(q\left(\frac{\Delta^{m} u_{k} x_{k}}{r}\right)\right)\right]^{p_{k}}\right)^{\frac{1}{H}} \leq 1 \quad n=1,2,3 . .\right\}
$$

Where $r=\frac{\rho}{\lambda}$. Since $|\lambda|^{p_{k}} \leq \max (1,|\lambda|)^{H}$, then $|\lambda|^{\frac{p_{k}}{H}} \leq\left(\max \left(1,|\lambda|^{H}\right)\right)^{\frac{1}{H}}$

Hence

$$
\begin{aligned}
g_{u}(\lambda x) \leq & \max (1,|\lambda|)^{H} \\
& \inf \left\{(r)^{\frac{p_{n}}{H}}:\left(\sum_{k=1}^{\infty} k^{-s}\left[M\left(q\left(\frac{\Delta^{m} u_{k} x_{k}}{r}\right)\right)\right]^{p_{k}}\right)^{\frac{1}{H}} \leq 1 n=1,2,3 . .\right\}
\end{aligned}
$$

and therefore $g_{u}(\lambda x)$ converges to zero where $g_{u}(x)$ converges to zero in

$$
\ell_{M}\left(\Delta^{m}, u, p, q, s\right) \text {. }
$$

Now suppose that $\lambda_{n} \rightarrow 0$ and $x \in \ell_{M}\left(\Delta^{m}, u, p, q, s\right)$. For arbitrary $\epsilon>0$. Let $\mathbb{N}$ be a positive integer such that

$$
\sum_{k=N+1}^{\infty} k^{-s}\left[M\left(q\left(\frac{\Delta^{m} u_{k} x_{k}}{\rho}\right)\right)\right]^{p_{k}}<\left(\frac{\epsilon}{2}\right)^{H}
$$

for some $\rho>0$. This implies that

$$
\left(\sum_{k=N+1}^{\infty} k^{-s}\left[M\left(q\left(\frac{\Delta^{m} u_{k} x_{k}}{\rho}\right)\right)\right]^{p_{k}}\right)^{\frac{1}{H}} \leq \frac{\epsilon}{2}
$$

Let $0<|\lambda|<1$, then using Remark 1.1 we get

$$
\sum_{k=N+1}^{\infty} k^{-s}\left[M\left(q\left(\frac{\Delta^{m} \lambda u_{k} x_{k}}{\rho}\right)\right)\right]^{p_{k}}<\sum_{k=N+1}^{\infty} k^{-s}\left[|\lambda| M\left(q\left(\frac{\Delta^{m} u_{k} x_{k}}{\rho}\right)\right)\right]^{p_{k}}<\left(\frac{\epsilon}{2}\right)^{H} .
$$


Since $M$ is continuous everywhere in $[0, \infty)$, then

$$
f(t)=\sum_{k=1}^{N} k^{-s}\left[M\left(q\left(\frac{\Delta^{m} t u_{k} x_{k}}{\rho}\right)\right)\right]^{p_{k}}
$$

is continuous at 0 . So there is $1>\delta>0$, such that $|f(t)|<\frac{\epsilon}{2}$ for $0<t<\delta$. Let $\mathrm{K}$ be such that $\left|\lambda_{n}\right|<\delta$ for $n>K$, then for $n>K$ we have

$$
\left(\sum_{k=1}^{\infty} k^{-s}\left[M\left(q\left(\frac{\Delta^{m} \lambda_{n} u_{k} x_{k}}{\rho}\right)\right)\right]^{p_{k}}\right)^{\frac{1}{H}}<\frac{\epsilon}{2}
$$

Since $0<\epsilon<1$ we have,

$$
\left(\sum_{k=1}^{\infty} k^{-s}\left[M\left(q\left(\frac{\Delta^{m} \lambda_{n} u_{k} x_{k}}{\rho}\right)\right)\right]^{p_{k}}\right)^{\frac{1}{H}}<1, \text { for } n>K
$$

If we take limit on $\inf \left\{\rho^{\frac{p_{n}}{H}}\right\}$ we get $g_{u}(\lambda x) \rightarrow 0$.

\section{Some Particular Cases}

We get the following sequence spaces from $\ell_{M}\left(\Delta^{m}, p, u, q, s\right)$ on giving particular values to $p, q, s$. Taking $p_{k}=1$ for all $k \in \mathbb{N}$

$$
\begin{aligned}
& \ell_{M}\left(\Delta^{m}, u, q, s\right)= \\
& \quad\left\{x \in \omega(X): \sum_{k=1}^{\infty} k^{-s}\left[M\left(q\left(\frac{u_{k} \Delta^{m} x_{k}}{\rho}\right)\right)\right]<\infty, s \geq 0 \text { for some } \rho>0\right\} .
\end{aligned}
$$

If $s=0$, then we have

$\ell_{M}\left(\Delta^{m}, u, p, q\right)=\left\{x \in \omega(X): \sum_{k=1}^{\infty}\left[M\left(q\left(\frac{u_{k} \Delta^{m} x_{k}}{\rho}\right)\right)\right]^{p_{k}}<\infty\right.$, for some $\left.\rho>0\right\}$.

If $p_{k}=1$ for all $k \in \mathbb{N}$, and $s=0$, then we have

$$
\ell_{M}\left(\Delta^{m}, u, q\right)=\left\{x \in \omega(X): \sum_{k=1}^{\infty}\left[M\left(q\left(\frac{u_{k} \Delta^{m} x_{k}}{\rho}\right)\right)\right]<\infty, \text { for some } \rho>0\right\} .
$$


If we take $s=0, q(x)=|x|$ and $X=\mathbb{C}$, then we have

$$
\ell_{M}\left(\Delta^{m}, u, p\right)=\left\{x \in \omega(X): \sum_{k=1}^{\infty}\left[M\left(\frac{\left|u_{k} \Delta^{m} x_{k}\right|}{\rho}\right)\right]^{p_{k}}<\infty, \text { for some } \rho>0\right\} .
$$

In addition to the above sequence spaces, we have $\ell_{M}\left(\Delta^{m}, u, p, q, s\right)=\ell_{M}(p)$ due to Prashar and Choudhry [16], on taking $u_{k}=1$ for all $k \in \mathbb{N}, m=0, s=$ 0 , and $q(x)=|x|$ and $X=\mathbb{C}$. If we take $u_{k}=1$ for all $k \in \mathbb{N}$, and $m=0$ we have $\ell_{M}\left(\Delta^{m}, p, u, q, s\right)=\ell_{M}(p, q, s)$, see [1].

Theorem 3.1. Let $0 \leq p_{k} \leq t_{k}<\infty$ for each $k \in \mathbb{N}$. Then:

(i) $\ell_{M}\left(\Delta^{m}, p, u, q\right) \subseteq \ell_{M}\left(\Delta^{m}, t, u, q\right)$

(ii) $\ell_{M}\left(\Delta^{m}, u, q\right) \subseteq \ell_{M}\left(\Delta^{m}, u, q, s\right)$

(iii) $\ell_{M}\left(\Delta^{m}, p, u, q\right) \subseteq \ell_{M}\left(\Delta^{m}, p, u, q, s\right)$

Proof. Let $x \in \ell_{M}\left(\Delta^{m}, p, u, q, s\right)$. Then there exist some $\rho>0$ such that

$$
\sum_{k=1}^{\infty}\left[M\left(q\left(\frac{u_{k} \Delta^{m} x_{k}}{\rho}\right)\right)\right]^{p_{k}}<\infty .
$$

This implies that $\left[M\left(q\left(\frac{u_{i} \Delta^{m} x_{i}}{\rho}\right)\right)\right] \leq 1$ for sufficiently large values of $i$, says that $i \geq k_{0}$ for some fixed $k_{o} \in \mathbb{N}$. Since $M$ is non-decreasing, we get

$$
\sum_{k=1}^{\infty}\left[M\left(q\left(\frac{u_{k} \Delta^{m} x_{k}}{\rho}\right)\right)\right]^{t_{k}}<\infty
$$

since

$$
\sum_{k \geq k_{0}}^{\infty}\left[M\left(q\left(\frac{u_{k} \Delta^{m} x_{k}}{\rho}\right)\right)\right]^{t_{k}} \leq \sum_{k \geq k_{0}}^{\infty}\left[M\left(q\left(\frac{u_{k} \Delta^{m} x_{k}}{\rho}\right)\right)\right]^{p_{k}}<\infty .
$$

Hence $x \in \ell_{M}\left(\Delta^{m}, u, t, q\right)$.

The proof of $(i i)$ and $(i i i)$ is trivial.

Theorem 3.2. Let $0<p_{k} \leq t_{k}<\infty$ for each $k$. Then $\ell_{M}\left(\Delta^{m}, u, p\right) \subseteq$ $\ell_{M}\left(\Delta^{m}, u, t\right)$.

Proof. Proof can be given by the same way as Theorem 3.1(i)

Theorem 3.3.

(i) If $0<p_{k} \leq 1$ for all $k \in \mathbb{N}$, then $\ell_{M}\left(\Delta^{m}, u, p, q\right) \subseteq \ell_{M}\left(\Delta^{m}, u, q\right)$ 
(ii) If $p_{k} \geq 1$ for all $k \in \mathbb{N}$, then $\ell_{M}\left(\Delta^{m}, u, q\right) \subseteq \ell_{M}\left(\Delta^{m}, u, p, q\right)$

Proof. ( $i$ ) If we take $t_{k}=1$ for all $k \in \mathbb{N}$, in Theorem 3.1(i), then $\ell_{M}\left(\Delta^{m}, u, p, q\right) \subseteq \ell_{M}\left(\Delta^{m}, u, q\right)$

(ii) f we take $p_{k}=1$ for all $k \in \mathbb{N}$, in Theorem 3.1(i), then $\ell_{M}\left(\Delta^{m}, u, q\right) \subseteq$ $\ell_{M}\left(\Delta^{m}, u, p, q\right)$

Proposition 3.4. For any two sequence $p=\left(p_{k}\right)$ and $t=\left(t_{k}\right)$ of positive real numbers and any two seminorms $q_{1}$ and $q_{2}$ we have $\ell_{M}\left(\Delta^{m}, u, p, q_{1}, r\right) \cap$ $\ell_{M}\left(\Delta^{m}, u, t, q_{2}, s\right) \neq \emptyset$ for all $m, n \in \mathbb{N} r, s>0$.

Proof. Since the Zero element belongs to $\ell_{M}\left(\Delta^{m}, u, p, q_{1}, r\right)$ and $\ell_{M}\left(\Delta^{m}, u\right.$, $\left.t, q_{2}, s\right)$, thus the intersection is nonempty.

Theorem 3.5. The sequence space $\ell_{M}\left(\Delta^{m}, u, p, q, s\right)$ is solid.

Proof. Let $\left(x_{k}\right) \in \ell_{M}\left(\Delta^{m}, u, p, q, s\right)$, i.e,

$$
\sum_{k=1}^{\infty} k^{-s}\left[M\left(q\left(\frac{\Delta^{m} u_{k} x_{k}}{\rho}\right)\right)\right]^{p_{k}}<\infty
$$

$\operatorname{Let}\left(\alpha_{k}\right)$ be sequence of scalar such that $\left|\alpha_{k}\right| \leq 1$, for all $k \in \mathbb{N}$. Then we have

$$
\sum_{k=1}^{\infty} k^{-s}\left[M\left(q\left(\frac{\Delta^{m} \alpha_{k} u_{k} x_{k}}{\rho}\right)\right)\right]^{p_{k}} \leq \sum_{k=1}^{\infty} k^{-s}\left[M\left(q\left(\frac{\Delta^{m} u_{k} x_{k}}{\rho}\right)\right)\right]^{p_{k}}<\infty .
$$

Hence $\left(\alpha_{k} x_{k}\right) \in \ell_{M}\left(\Delta^{m}, u, p, q, s\right)$ for all sequences of scalar $\left(\alpha_{k}\right)$ with $\left|\alpha_{k}\right| \leq$ 1 for all $k \in \mathbb{N}$, whenever $\left(x_{k}\right) \in \ell_{M}\left(\Delta^{m}, u, p, q, s\right)$. Therefore the space $\ell_{M}\left(\Delta^{m}, u, p, q, s\right)$ is solid sequence space.

\section{Corollary 3.6.}

(i) Let $\left|u_{k}\right| \leq 1$ for all $k \in \mathbb{N}$. Then $\ell_{M}\left(\Delta^{m}, p, q, s\right) \subseteq \ell_{M}\left(\Delta^{m}, u, p, q, s\right)$

(ii) Let $\left|u_{k}\right| \geq 1$ for all $k \in \mathbb{N}$. Then $\ell_{M}\left(\Delta^{m}, u, p, q, s\right) \subseteq \ell_{M}\left(\Delta^{m}, p, q, s\right)$

Proof. Proof is trivial.

Theorem 3.7. Let $M, M_{1}$ and $M_{2}$ be an orlicz function and $s, s_{1}$ and $s_{2}$ be non negative real numbers. Then we have:

(i) Let $\ell_{M_{1}}\left(\Delta^{m}, u, p, q, s\right) \cap \ell_{M_{2}}\left(\Delta^{m}, u, p, q, s\right) \subseteq \ell_{M_{1}+M_{2}}\left(\Delta^{m}, u, p, q, s\right)$

(ii) If $s_{1} \leq s_{2}$, then $\ell_{M}\left(\Delta^{m}, u, p, q, s_{1}\right) \subseteq \ell_{M}\left(\Delta^{m}, u, p, q, s_{2}\right)$ 
Proof.(i) From (1) we have

$$
\begin{gathered}
k^{-s}\left[\left(M_{1}+M_{2}\right)\left(q\left(\frac{\Delta^{m} u_{k} x_{k}}{\rho}\right)\right)\right]^{p_{k}} \\
=k^{-s}\left[M_{1}\left(q\left(\frac{\Delta^{m} u_{k} x_{k}}{\rho}\right)\right)+M_{2}\left(q\left(\frac{\Delta^{m} u_{k} x_{k}}{\rho}\right)\right)\right]^{p_{k}} \\
\leq D k^{-s}\left[M_{1}\left(q\left(\frac{\Delta^{m} u_{k} x_{k}}{\rho}\right)\right)\right]^{p_{k}}+D k^{-s}\left[M_{2}\left(q\left(\frac{\Delta^{m} u_{k} x_{k}}{\rho}\right)\right)\right]^{p_{k}}
\end{gathered}
$$

Let $x \in \ell_{M_{1}}\left(\Delta^{m}, u, p, q, s\right) \cap \ell_{M_{2}}\left(\Delta^{m}, u, p, q, s\right)$, when adding the above inequality from $k=1$ to $\infty$, we get $x \in \ell_{M_{1}+M_{2}}\left(\Delta^{m}, u, p, q, s\right)$.

(ii) Let $s_{1} \leq s_{2}$ and $x \in \ell_{M}\left(\Delta^{m}, u, p, q, s_{1}\right)$. Since $k^{-s_{2}} \leq k^{-s_{1}}$, we have $x \in \ell_{M}\left(\Delta^{m}, u, p, q, s_{2}\right)$

This completes the proof.

\section{References}

[1] Biktas, C and Altin, Y., The sequence space $\ell_{M}(p, q, s)$ on seminormed spaces, Indian J. Pure Appl. Math. 34 (2003), 529-534.

[2] Bilgen, T., The sequence space $\ell(p, f, q, s)$ on seminormed spaces, Bull. Calcutta Math. Soc. 86 (1994), 295-304.

[3] ET, M., On some topological properties of generalized difference sequence spaces, Int. J. Math. Math. Sci. 24 (2000), 785-791.

[4] ET, M and Çolack, R., On some generalized difference sequence spaces, Soochow J. Math. 21 (1995), 377-386.

[5] ET, M and Nuray, F., $\Delta^{m}$-statistical convergence, Indian J. Pure Appl. Math. 32 (2001), 961-969.

[6] Kamthan ,P. K and Gupta M., Sequence spaces and series. Lecture Notes in Pure and Appl .Math. 65, Marcel Dekker, Inc. New York, 1981.

[7] Kizmaz, H., On certain sequence spaces, Canad. Math. Bull. 24 (1981), 169-176.

[8] Krasnoselskii, M. A and Rutickii,Y.B., Convex functions and orlicz spaces, Groningen, Netherlands, 1961 
[9] Lindberg, K., On subspaces of orlicz sequence spaces, Studia Math. 45 (1973), 119-146.

[10] Lindenstrauss, J and Tzafriri, L., On orlicz sequence spaces, Israel J. Math. 10 (1971), 379-390.

[11] Maddox, I. J., Sequence spaces defined by a modulus, Math. Proc. Cambridge Philos.Soc. 100 (1986), 161-166.

[12] Maddox, I. J., Elements of functional Analysis, Cambridge Univ. Press (1970).

[13] Malkosky, E and Prashar, S. D., Matrix transformations in spaces of bounded convergent and difference sequences of order $m$, Analysis (Munich) 17 (1997), 87-97.

[14] Mursaleen, M., Generalized spaces of difference sequences, J. Math. Anal. Appl. 203 (1996), 738-745.

[15] Nakno. H., Concave modulars, J. Math. Soc. Japan 5 (1953), 29-49.

[16] Prashar, S. D and Chouddry, B., Sequence spaces defined by orlicz function, Indian J. Pure Appl. Math. 25 (1994), 419-428.

[17] Ruckle, W. H., FK spces in which the sequence of coordinates vectors is bounded, Canad. J. Math. 25 (1973), 973-978.

[18] Tripathy, B. C., On generalized difference paranormed statistically convergent sequences, Indian J. Pure Appl. Math. 35 (2004), 655-663.

[19] Tripathy, B. C., Generalized difference paranormed statistically convergent sequences defined by orlicz function in locally convex spaces, Soochow J. Math. 30 (2004), 431-446.

[20] Tripathy. B.C, ET. M and M. Altin. Y., Genaralized difference sequence spaces defined by orlicz function in a locally convex space. J. Anal. Appl. 1 (2003), 175-192.

[21] Tripathy, B. C and Mahanta. S., On a class of sequences related to the $\ell^{p}$ spaces defined by orlicz function, Soochow J. Math. 29 (2003), 379-391.

[22] Tripathy, B. C and Mahanta. S., On a class of generalized lacunary difference sequence spaces defined by orlicz functions, Acta. Math. Appl. Sinica (English Ser.) 20 (2004), 231-238. 
[23] W.L.C., Sargent., Some sequence spaces related to the $\ell^{p}$ spacess, J. London Math. Soc. 35 (1960), 161-171

[24] Yosidak, K., Functional Analysis, Springer- Verlag, Berlian- Heidelberg Newyork., (1971) 
